\title{
A TILTING WATER METER FOR PURPOSES OF EXPERIMENT.
}

By J. C. Hoadley, Boston, Mass.

Having had to make, in the course of the year, a great number of experiments in pumping water filtered through sand under various prearranged conditions, in quantities ranging from little more than one gallon in an hour to eight or ten gallons in a minute, the writer soon found that all commercial water meters were wholly unsuitable for his purposes. They were liable to obstruction by sand, inaccurate at best, wildly inaccurate at very low speeds, and difficult to read at high speeds. In addition to this, the readings were at all times insusceptible of verification and of permanent record.

Not having found, after scarch made, any account of a suitable

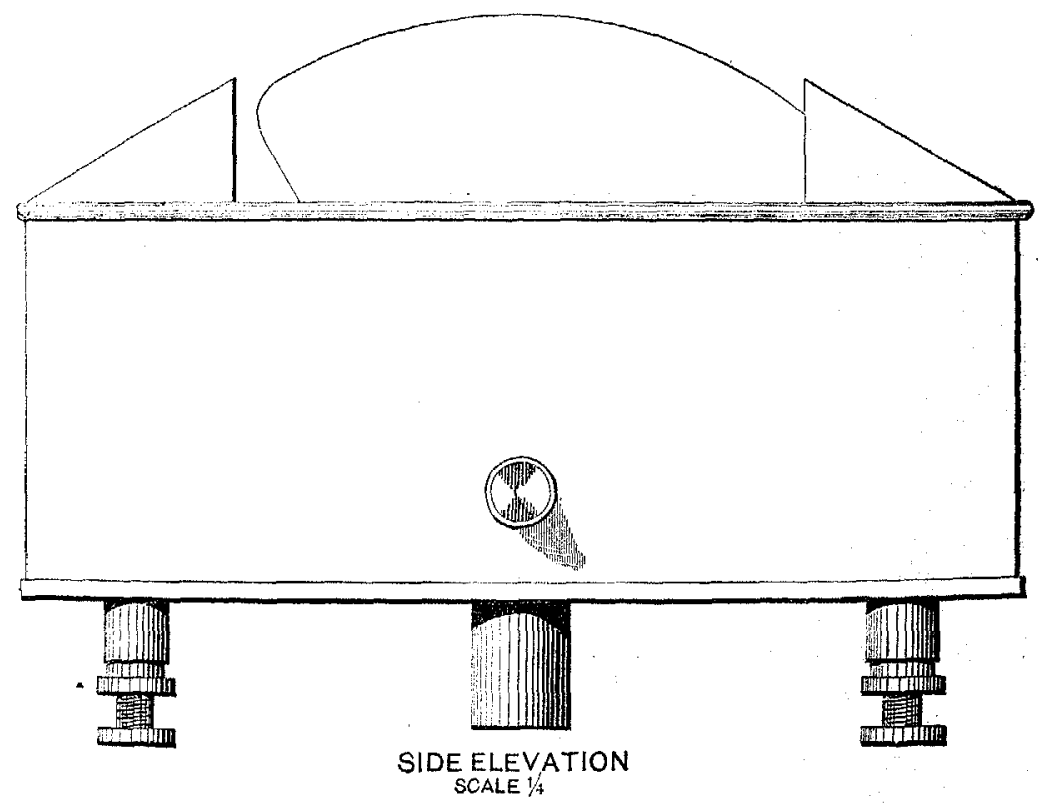

instrument, it became necessary to devise one, and without presuming that this is wholly new or unknown to all the members of this society, drawings and a description of it are presented as a matter of record for what it is worth.

Wrote No. Vor. CXVII.-(Throd SerIes, Vol, lxxxvii.) 
There was required an instrument which would measure and record with all possible accuracy, and without liability to important error, the quantity of water flowing in a continuous, but pulsating, and sometimes variable, stream, in accurately ascertained intervals of time. As construeted and used the instrument is very simple and inexpensive, and is clearly shown in the acompanying drawings.

Two V-shaped cups, ach embracing an angle of $60^{\circ}$, are joined together by a common side, which is, in fact, a mere partition between them, so that the two cups together embrace an angle of $120^{\circ}$. This double cup is supported in a case upon pivots directly under the partition, tuming in hollow, arjustable screws in nuts attached to the case, one on cach side. When one of the outside plates of the double-y cup is in a horizontal position, supported in that position ly two cork stops on which it rests, the partition between the two $V$-cups makes an angle of $30^{\circ}$ with the vertical, and the outside plate of the upright cup makes an angle also of $30^{\circ}$ with the vertical, but at al little greater

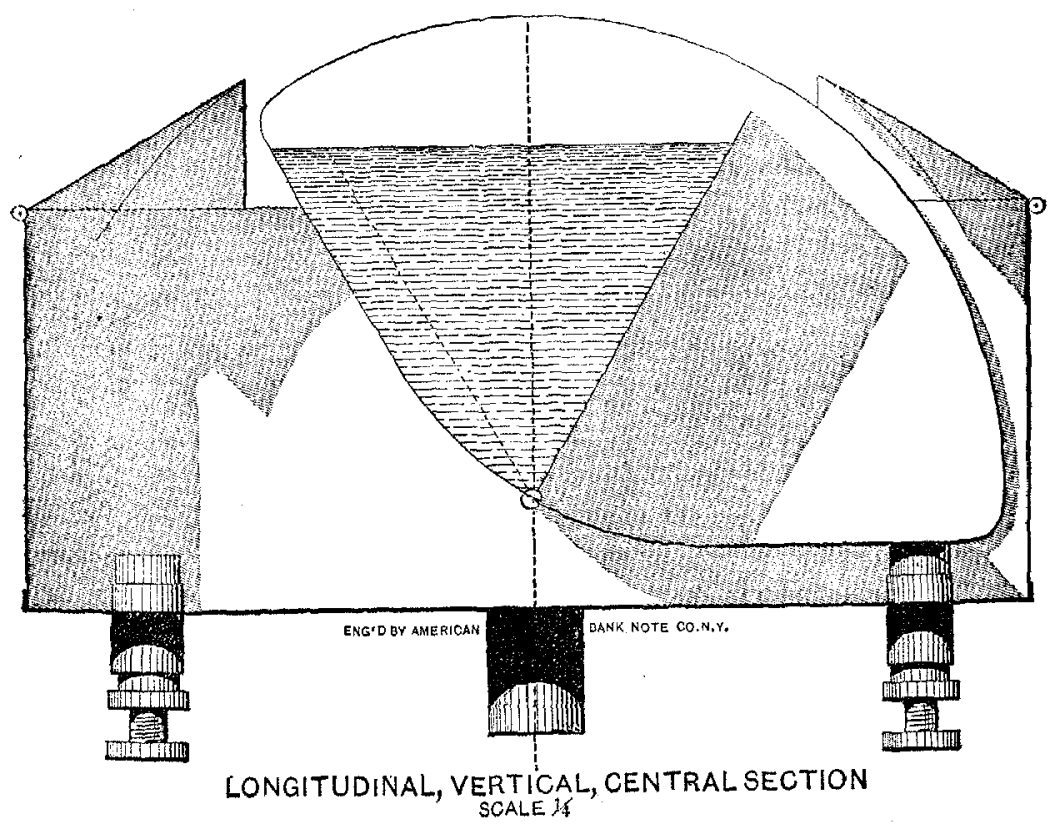

distance horizontally from a vertical plane passing through the axis of the pivots, on account of the curve by which the outside plate is joined 
to the partition and to the outside plate of the prostrate cup at its lower end. But for this greater horizontal width on the outer side there would be no tendency to tip-the upright cup would simply fill up and overflow, and there would be an end. But as the water rises in the upright cup, the prism of water outside of a plane passing through the axis of the pivots and making an angle of $30^{\circ}$ with the vertical, acquires constantly increasing preponderance over the equipoise of the wedge-shaped body of water bounded by planes, each making an angle of $30^{\circ}$ with the vertical, and intersecting in the axis of the

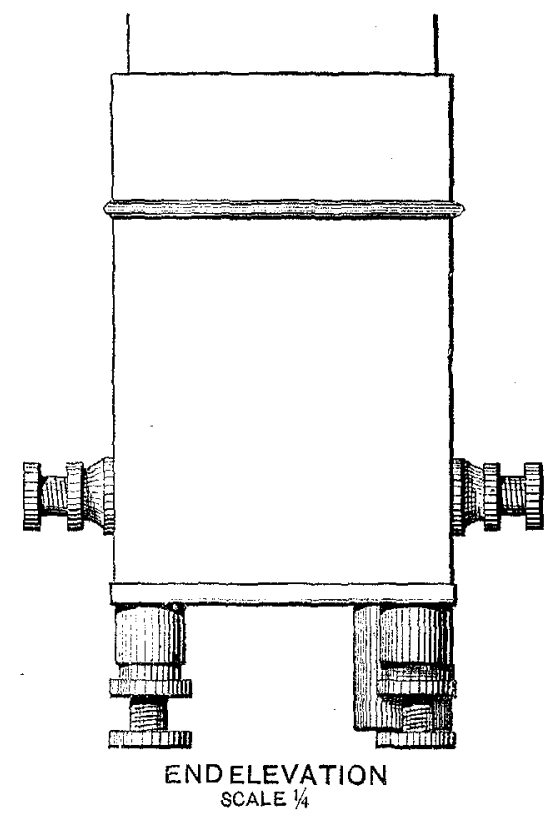

pivots. When this preponderance becomes sufficient to overcome the mechanical advantage of the prostrate cup itself over the upright cup -an advantage due to its greater leverage with equal weight-the cup will tilt, the water in the upright cup, nearly filling it, will be poured out into the case to run away through its spout, and the now empty cup, lying prone on its cork stops, will become the prostrate cup, with the greater leverage-its center of gravity being at the greater horizontal distance from the axis of the pivot. The other cup, now upright, will be filled in its turn and repeat the tilting process, and so on alternately as long as the stream flows into the cups.

The tip is very sudden, and is made with considerable force. A. 
light spring of shect brass attached to the case in the middle of its length by a picce of wood which insulates it is connected by a binding post with one pole of a battery, and the case itself is in like manner connected with the other pole. A bit of sheet brass soldered to the outside of the tilting double cup, directly opposite the partition and above the case, forming a sort of cam, comes into contact with the spring in passing. This completes the circuit interrupted by the block of wood which supports the spring, and records each tip by a dot or

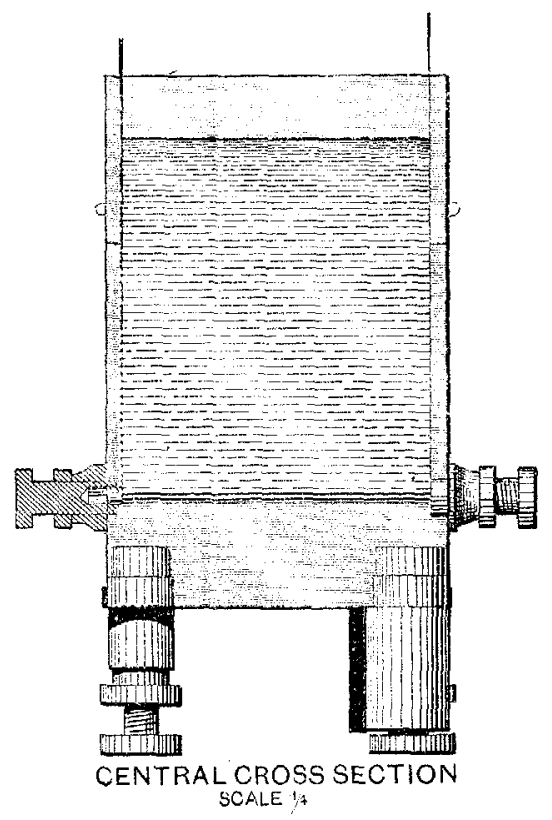

short dash. 'The cam does not come into contact with the spring until the tilting cup has acquired considerable momentum, so that the tilting is not sensibly retarded.

In designing this instrument the weight per square inch of the sheet tin selected for it was first ascertained. The whole of the double cup, ends, sides and partition lying on each side of a vertical plane, passing through the axis of the pivots, was then divided into simple geometrical figures, the center of gravity of each figure was found, and the horizontal distance of the common center of gravity of each portion from the vertical was computed. The weight of each portion lying on each side of the vertical was also computed, and multiplied by the corresponding horizontal distance of each center of gravity, which gave, of 
course, the static moment of that portion of the double cup on each side of a vertical plane passing through the axis of the pivots.

The difference of these static moments was the preponderance of the prostrate over the upright cup-both empty-to be overcome by the water in the upright cup when full to the tilting point, and this was computed in a similar nanner. A slight adjustment of the cork stops sufficed to compensate for the inaccuracy of these calculations.

This leads me to speak of the matter of adjustment by means of the cork stops. I actually used a mere socket, like a short candlestick, to hold each cork, entirely inside of the case, which was plain on the bottom. In the drawings will be seen four long sockets, projecting below as well as above the bottom of the case, with adjusting screws and eheck nuts. The adjustable corks are held in short sockets, with thick

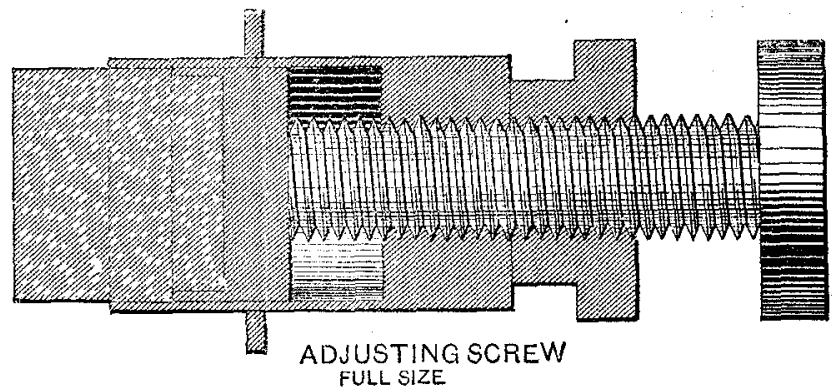

bottoms to rest on the adjusting screws, heavy enough to keep the corks from floating away. This device is untried, but $I$ think it quite practicable and no less desirable.

I had two of these meters, substantially alike, and adjusted them by flowing water through them into a tub placed on scales, counting the tips, and taking the weight and temperature of the water. I then placed one of the meters directly over the other, supported upon the tub by a suitable frame, and flowed a stream through both, the electric register recording the tips, and the scales accounting for the quantity of water. The first tip of the upper meter did not fill the lower meter to the tipping point by about $\frac{1}{16}$ inch, a little water adhering to the cup and case of the first meter, but the second tip or the first was instantly followed by the first tip of the second, and so on until the tub was filled. A repetition of this experiment gave substantially the same results -61 tips in $202 \frac{7}{8}$ pounds $=3.33$ pounds per tip, and at 80:F., 0.4 gallon per tip-231 cubic inches per gallon.

The electric register also recorded upon the same $\frac{1}{2}$-inch strip of 
paper the beats of a seconds pendulum, and, when two pumps were used, the strokes of the pumps and the tips of each meter. It was also easy to note the tips by the ear while watching the second band of a horse-timer, by which means the interval of time between tips could easily be observed to quarters of a second. This is not an instrument of precision. It is, perhaps, a little more accurate than a gallon measure at all practicable speeds; at slow speeds probably decidedly more accurate, and, with proper care, no considemble error is likely to occur. When operated very rapidly the swaying of the surface tends to accelerate or retard in a small degree the tipping of a cup nearly full, but there is no tendency to accumulation in such errors, which, therefore, may be presumed to balance each other, at least in some degree. The sloping covers at the ends of the ase were an afterthought to prevent spattering, and were kept a sufficient distance apart to admit of taking out and replacing the tilting double cups. The curved wings: under these sloping cups were a second afterthought for the same purpose, and, as it stands, the case arrests all water-there is no spattering. Of course, a suitable funncl is generally desirable to collect the water from a pump and to convey it into the cup in a stream as steady as possible, and vertically over the axis of the pivots. It is also necessary that the meter should be level, both when adjusted and when in. use.

This instrument substantially weighs the water of a flowing stream, and may possibly prove useful, if strongly and delicately made, nicely adjusted and suitably porportioned to the quantity to be recorded, for keeping the record of water used during tests of steam engines and boilers. It is respectfully submitted to the profession for what it is worth.

Distillation of Benzine.-J. Chevalier, a dyer and scourer, of Toulon, employs benzine in cleaning his articles. The benzine soon becomes charged with foreign matters, is discolored, exhales a disagreeable odor, evaporates very slowly and loses its cleansing properties, so. that it must be replaced by a new supply. Thinking that it might. be possible to make a great saving by distilling the deteriorated benzine, he obtained remarkable results. The distillation gave a product which was limpid, much purer than the ordinary commercial benzine, ten per cent. lighter, and much more efficient in its action.-Chron. Industr., April 8, 1883. 